\title{
Strong Equality of MAJORITY Domination Parameters
}

\author{
J. Joseline Manora ${ }^{1}$ and B. John ${ }^{2}$ \\ ${ }^{I} P G \&$ Research Department of Mathematics, T.B.M.L College, Porayar, Nagai Dt., Tamilnadu, India. \\ ${ }^{2}$ Department of Science and Humanities, E.G.S.PillayEngineering College, Nagappattinam.
}

\begin{abstract}
We study the concept of strong equality of majority domination parameters. Let $P_{1}$ and $P_{2}$ be properties of vertex subsets of a graph, and assume that every subset of $V(G)$ with property $P_{2}$ also has property $P_{1}$. Let $\psi_{1}(G)$ and $\psi_{2}(G)$, respectively, denote the minimum cardinalities of sets with properties $P_{1}$ and $P_{2}$, respectively. Then $\psi_{1}(G) \leq \psi_{2}(G)$. If $\psi_{1}(G)=\psi_{2}(G)$ and every $\psi_{1}(G)$-set is also a $\psi_{2}(G)$-set , then we say $\psi_{1}(G)$ strongly equals $\psi_{2}(G)$, written $\psi_{1}(G) \equiv \psi_{2}(G)$. We provide a constructive characterization of the trees $T$ such that $\gamma_{M}(T) \equiv i_{M}(T)$, where $\gamma_{M}(T)$ and $i_{M}(T)$ are majority domination and independent majority domination numbers, respectively.
\end{abstract}

Keywords: Domination number, Majority domination number, Independent majority domination number, Strong equality. 2010 Mathematics Subject Classification: 05C69

\section{Introduction:}

By a graph $\mathrm{G}$, we mean a finite, simple and undirected. Let $\mathrm{G}$ be a graph with $\mathrm{p}$ vertices and $\mathrm{q}$ edges. For a vertex $v \in V(G)$, the open neighborhood of $v, N_{G}(v)$ is the set of vertices adjacent to $v$ and the closed neighborhood $N_{G}[v]=N_{G}(v) \bigcup\{v\}$. Other graph theoretic terminology not defined here can be found in [6]. In [6], A set $S \subseteq V$ of vertices in a graph $G=(V, E)$ is a dominating set if every vertex $v \in V$ is either an element of $\mathrm{S}$ or is adjacent to an element of $\mathrm{S}$. A dominating set $\mathrm{S}$ is called a minimal dominating set if no proper subset of $\mathrm{S}$ is a dominating set. The minimum cardinality of a minimal dominating set is called the domination number $\gamma(G)$ and the maximum cardinality of a minimal dominating set is called the upper domination number $\Gamma(G)$ in a graph G. A set $S \subseteq V$ of vertices in a graph $\mathrm{G}$ is called an independent set if no two vertices in $\mathrm{S}$ are adjacent. An independent set $\mathrm{S}$ is called a maximal independent set if any vertex set properly containing $\mathrm{S}$ is not independent. The minimum cardinality of a maximal independent set is called the lower independence number and also independent domination number and the maximum cardinality of a maximal independent set is called the independence number in a graph $\mathrm{G}$ and it is denoted by $i(G)$ and $\beta_{o}(G)$ respectively.

\section{Definition 1.1[3]:}

A subset $S \subseteq V(G)$ of vertices in a graph $G$ is called majority dominating set if at least half of the vertices of $V(G)$ are either in $S$ or adjacent to the vertices of $S$. i.e., $|N[S]| \geq\left\lceil\frac{p}{2}\right\rceil$. A majority dominating set $S$ is minimal if no proper subset of $S$ is a majority dominating set of G. The majority domination number $\gamma_{M}(G)$ of a graph $\mathrm{G}$ is the minimum cardinality of a minimal majority dominating set in $\mathrm{G}$. The upper majority domination number $\Gamma_{M}(G)$ is the maximum cardinality of a minimal majority dominating set of a graph G. This parameter has been studied by Swaminathan V and JoselineManora J. 


\section{Definition 1.2[2]:}

A set $\mathrm{S}$ of vertices of a graph $\mathrm{G}$ is said to be a majority independent set if it induces a totally disconnected subgraph with $|N[S]| \geq\left\lceil\frac{p}{2}\right\rceil$ and $\left|p_{n}[v, S]\right|>|N[S]|-\left\lceil\frac{p}{2}\right\rceil$ for every $v \in S$. If any vertex set $S^{\prime}$ properly containing $\mathrm{S}$ is not majority independent then $\mathrm{S}$ is called maximal majority independent set.The maximum cardinality of a maximal majority independent set of $\mathrm{G}$ is called majority independence number of $\mathrm{G}$ and it is denoted by $\beta_{M}(G)$. A $\beta_{M}$-set is a maximum cardinality of a maximal majority independent set of $\mathrm{G}$. This parameter is introduced by Swaminathan. V and JoselineManora. J.

\section{Definition 1.3[1]:}

A majority dominating set $\mathrm{D}$ of a graph $G=(V, E)$ is called an independent majority dominating (IMD) set if the induced subgraph $<\mathrm{D}>$ has no edges. The minimum cardinality of a maximal majority independent set is called lower majority independent set of $\mathrm{G}$ and it is also called independent majority domination number of $\mathrm{G}$, denoted by $i_{M}(G)$.

If the degree of a vertex $v$ satisfies $d(v) \geq\left\lceil\frac{p}{2}\right\rceil-1$, then the vertex $v \in V(G)$ is called a majority dominating vertex of $\mathrm{G}$.

\section{Strong equality of Majority domination Parameters.}

Definition 2.1[5]:

Let $P_{1}$ and $P_{2}$ be properties of vertex subsets of a graph, and assume that every subset of $V(G)$ with property $P_{2}$ also has property $P_{1}$. Let $\psi_{1}(G)$ and $\psi_{2}(G)$, respectively, denote the minimum cardinalities of sets with properties $P_{1}$ and $P_{2}$, respectively. Then $\psi_{1}(G) \leq \psi_{2}(G)$ and every $\psi_{1}(G)$-set is also a $\psi_{2}(G)$ set, then we say $\psi_{1}(G)$ strongly equals $\psi_{2}(G)$, written $\psi_{1}(G) \equiv \psi_{2}(G)$.

\section{Definition 2.2:}

Let $G$ be any graph with $p$ vertices. Let $\gamma_{M}(G)$ and $i_{M}(G)$ be the majority domination number and independent majority domination number of a graph $G$. Then $\gamma_{M}(G)$ and $i_{M}(G)$ are strongly equal for $G$ if $\gamma_{M}(G)=i_{M}(G)$ and every $\gamma_{M}(G)$-set is an $i_{M}(G)$-set. It is denoted by $\gamma_{M}(G) \equiv i_{M}(G)$.

\section{Example 2.3:}

Take $j=2, \quad p=22 j=44 . D=\left\{u_{1,1}^{\prime}, u_{2,1}^{\prime}, u_{3,1}^{\prime}, u_{4,1}^{\prime}, u_{1,1}^{\prime \prime}, u_{2,1}^{\prime}\right\} . \quad \gamma_{M}\left(G_{j}\right)=|D|=6$. Since all vertices in $\mathrm{D}$ are independent, $i_{M}\left(G_{j}\right)=|D|=6 . \quad \therefore \gamma_{M}\left(G_{j}\right) \equiv i_{M}\left(G_{j}\right)=3 j, j=2$. Where as $\gamma(G)=i(G)=8 j=16, j=2$. 


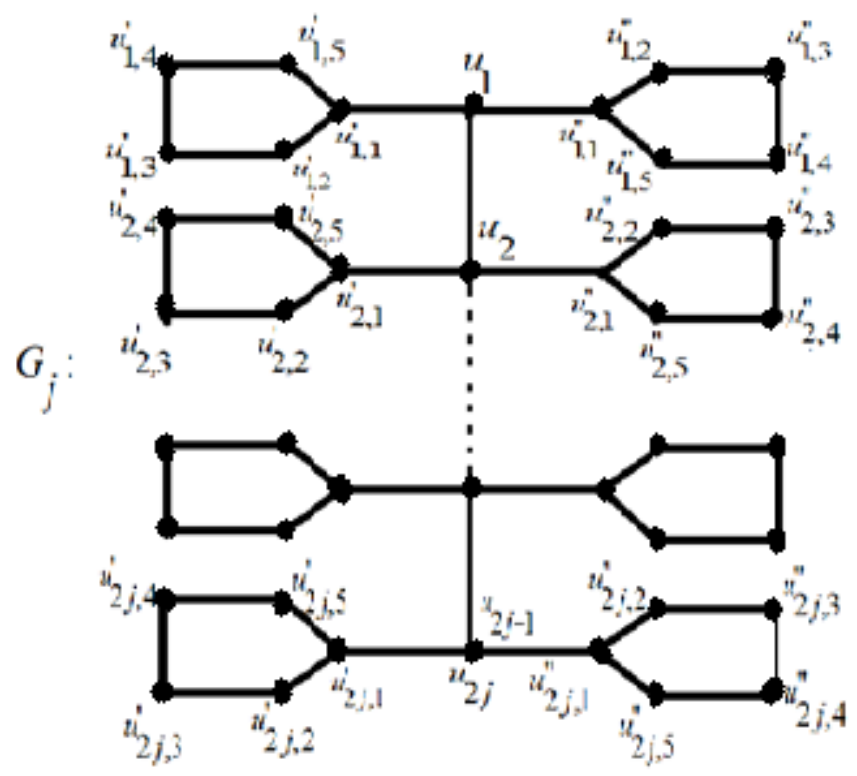

Fig ( $)$

\section{Observations 2.4:}

1. $\gamma_{M}\left(G_{j}\right)<\frac{\left|\gamma\left(G_{j}\right)\right|}{2} \Rightarrow 3 j<\frac{8 j}{2}=4 j$, where $G_{j}$ is in Fig (i).

2. When $j=1, \gamma_{M}\left(G_{j}\right)=3=i_{M}\left(G_{j}\right)$.

When $j=2, \gamma_{M}\left(G_{j}\right)=6=i_{M}\left(G_{j}\right)$.

When $j=3, \gamma_{M}\left(G_{j}\right)=9=i_{M}\left(G_{j}\right)$.

In general, for $G_{j}, \gamma_{M}\left(G_{j}\right) \equiv i_{M}\left(G_{j}\right)=3 j, \quad j=1,2, \ldots$

We can extend this graph by applying values to $j=2,3,4, \ldots$. Then we obtain $\gamma_{M}\left(G_{j}\right)=3 j=i_{M}\left(G_{j}\right)$. Also, every $\gamma_{M}$-set is an $i_{M}$-set of $G_{j}$. Hence $\gamma_{M}\left(G_{j}\right) \equiv i_{M}\left(G_{j}\right)$.

\section{Example 2.5:}

The graph $G$ is obtained from disjoint copies of $p_{5}$ by joining a central vertex of one $p_{5}$ to the central vertices of the remaining graphs $p_{5}$.

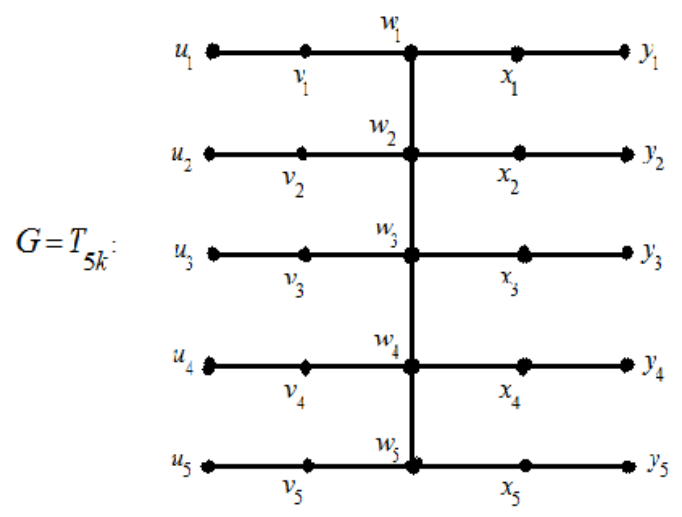

Fig (ii) 
When $k=5, p=25 . \quad D_{1}=\left\{w_{2}, w_{3}, w_{4}, w_{5}\right\} . \quad D_{1}$ dominates $\left\lceil\frac{p}{2}\right\rceil=13$ vertices. $\quad \therefore \gamma_{M}(G)=\left|D_{1}\right|=4$. $\therefore$ This $\gamma_{M}$-set $D_{1}$ is not an $i_{M}$-set of $G . D_{2}=\left\{w_{2}, w_{3}, v_{3}, v_{5}\right\} \Rightarrow i_{M}(G)=\left|D_{2}\right|=4$. But $D_{2}$ is a $\gamma_{M}$-set which is also an $i_{M}$-set. Here, $D_{1}$ and $D_{2}$ are minimal majority dominating set for $G \cdot \gamma_{M}(G)=i_{M}(G)=4$. Hence every $\gamma_{M}$-set is not an $i_{M}$-set for $G \therefore \gamma_{M}(G)$ is not $\equiv i_{M}(G)$, if $G=T_{5 k}, k=5$. Ingeneral, for any value of $k$, if $\gamma_{M}(G)$ is not $\equiv i_{M}(G)$, if $G=T_{5 k}, k=5$.

\section{Observations 2.6:}

1. If $\gamma_{M}(G)=1$ then $\gamma_{M}(G) \equiv i_{M}(G)$.

2. If $G$ has a full degree vertex then $\gamma_{M}(G) \equiv i_{M}(G)$.

3. For Corona graphs $G, \gamma_{M}(G) \equiv i_{M}(G)$, if $G=\left(C_{p} o K_{1}\right)$ and $G=\left(P_{p} o K_{1}\right)$.

4. $\gamma_{M}(G) \equiv i_{M}(G)$ if $\mathrm{G}=$ Caterpillar, with exactly one pendant.

5. $\gamma_{M}(G) \equiv i_{M}(G)$ if $G=m K_{2}$.

6. If $G$ is Grotzsch graph, then $\gamma_{M}(G) \equiv i_{M}(G)$.

7. For Tutte graph $G$ with $p=46, q=69 \cdot \gamma_{M}(G)=i_{M}(G)=6$.

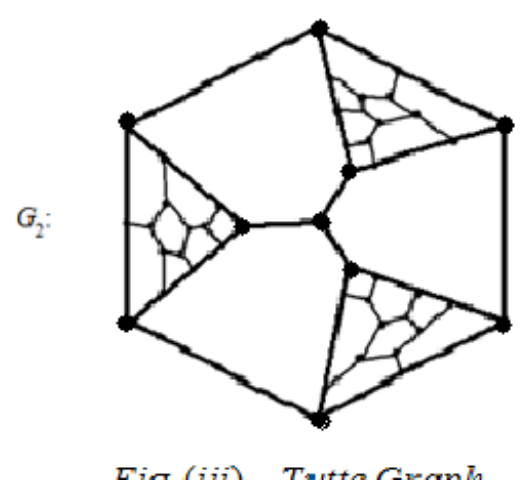

Fig (iii). Tutte Graph

Every $\gamma_{M}(G)$-set is an $i_{M}(G)$-set. Hence $\gamma_{M}(G) \equiv i_{M}(G)$.

8. For a Grinberg graph $\mathrm{G}$ with $p=46, q=69$, then $\gamma_{M}(G) \equiv i_{M}(G)$.

9. For a Petersen graph $\mathrm{P}, \gamma_{M}(P)$ is not strongly equal to $i_{M}(P)$.

10. For all Hajos graph $\mathrm{H}$ with $\mathrm{p}$ vertices,

where $p=\frac{n(n+1)}{2}, n=3,4 \Rightarrow p=6,10$, then $\gamma_{M}(H) \equiv i_{M}(H)$.

But if $n=5$ and $p=15$, then $\gamma_{M}(H)$ is not strongly equal to $i_{M}(H)$.

\section{Proposition 2.7:}

For the path $P_{p}$ and cycle $C_{p}$,

1. $\gamma_{M}\left(P_{6 k}\right) \equiv i_{M}\left(P_{6 k}\right)=\gamma_{M}\left(C_{6 k}\right) \equiv i_{M}\left(C_{6 k}\right)=k, k=1,2,3, \ldots$

2. $\gamma_{M}\left(P_{6 k+3}\right) \equiv i_{M}\left(P_{6 k+3}\right)=\gamma_{M}\left(C_{6 k+3}\right) \equiv i_{M}\left(C_{6 k+3}\right)=k+1, k=0,1,2, \ldots$

3. $\gamma_{M}\left(P_{6 k+4}\right) \equiv i_{M}\left(P_{6 k+4}\right)=\gamma_{M}\left(C_{6 k+4}\right) \equiv i_{M}\left(C_{6 k+4}\right)=k+1, k=0,1,2, \ldots$ 
4. $\gamma_{M}\left(P_{6 k+5}\right) \equiv i_{M}\left(P_{6 k+5}\right)=\gamma_{M}\left(C_{6 k+5}\right) \equiv i_{M}\left(C_{6 k+5}\right)=k+1, k=0,1,2, \ldots$, but

5. $\gamma_{M}\left(P_{6 k+1}\right)$ is not $\equiv i_{M}\left(P_{6 k+1}\right)=\gamma_{M}\left(C_{6 k+1}\right)$ is not $\equiv i_{M}\left(C_{6 k+1}\right)=k+1, k=0,1,2, \ldots$

6. $\gamma_{M}\left(P_{6 k+2}\right)$ is not $\equiv i_{M}\left(P_{6 k+1}\right)=\gamma_{M}\left(C_{6 k+2}\right)$ is not $\equiv i_{M}\left(C_{6 k+1}\right)=k+1, k=0,1,2, \ldots$

\section{Proposition 2.8[4]:}

For any graph $G, \gamma_{M}(G)=1$ if and only if $G$ has a majority dominating vertex.

\section{Proposition 2.9:}

$\gamma_{M}(G) \equiv i_{M}(G)=1$ if and only if $G$ has a majority dominating vertex.

III. Trees $T$ with $\gamma_{M}(T) \equiv i_{M}(T)$

Our aim in this section is to give a constructive characterization for the trees $T$ having $\gamma_{M}(T) \equiv i_{M}(T)$. For this purpose, we first prove two lemmas.

Lemma 3.1:

Let $w$ be a vertex of a tree $T_{w}$ such that every leaf of $T_{w}$, except possibly for $w$ itself, is at distance two from $w$. Let $S_{w}$ be the set of support vertices of $T_{w}$. Let $y$ be a pendant vertex of a non-trivial tree $T_{y}$. Let $T$ be obtained from $T_{w} \cup T_{y}$ by adding theedge $w y$. Then $\gamma_{M}(T)=\gamma_{M}\left(T_{y}\right)+1$.

Proof: Let $T=T_{y} \cup T_{w}$ and $y$ be a pendant vertex of $d(y)=2, y \in T$. Let $\gamma_{M}\left(T_{y}\right)$ be a majority domination number of $T_{y}$. Since $w$ is a majority dominating vertex of $T_{w}, \gamma_{M}\left(T_{y}\right)$-set can be extended to a majority dominating set of $T$ by adding the vertex $w \in T_{w} \therefore \gamma_{M}(T) \leq \gamma_{M}\left(T_{y}\right)+1$.

Claim: $\gamma_{M}(T) \geq \gamma_{M}\left(T_{y}\right)+1$. Let $D$ be a $\gamma_{M}$-set of $T$. Then $D_{y}=D \cap V\left(T_{y}\right)$ and $D_{w}=D \cap V\left(T_{w}\right)$. Since $T_{W}$ has a majority dominating vertex $w .\left|D_{w}\right|=|\{w\}|=1$.

Since $D$ is a $\gamma_{M}$-set of $T, D_{y} \quad$ is a majority dominating set of $T_{y}$. Then $\gamma_{M}\left(T_{y}\right) \leq\left|D_{y}\right| \leq\left|D-D_{w}\right| \Rightarrow \gamma_{M}\left(T_{y}\right) \leq|D|-1=\gamma_{M}(T)-1 \Rightarrow \gamma_{M}\left(T_{y}\right)+1 \leq \gamma_{M}(T)$.

Hence, $\gamma_{M}(T)=\gamma_{M}\left(T_{y}\right)+1$.

\section{Lemma 3.2:}

Let $T_{w}, T_{y}$, and $T$ be defined as in the statement of Lemma (3.1). Then $\gamma_{M}(T) \equiv i_{M}(T)$ if and only if $\gamma_{M}\left(T_{y}\right) \equiv i_{M}\left(T_{y}\right)$.

Proof: Suppose $\gamma_{M}(T) \equiv i_{M}(T) \quad \ldots \ldots .(1)$. Let $D_{y}$ be a $\gamma_{M}\left(T_{y}\right)$-set. Then $D_{y} \cup\{w\}$ is a majority dominating set of $T$ of cardinality $\gamma_{M}\left(T_{y}\right)+1$. Then by lemma (3.1), $\gamma_{M}(T)=\gamma_{M}\left(T_{y}\right)+1$. Therefore $D_{y} \cup\{w\}$ is a $\gamma_{M}(T)$-set and by (1), it is a $i_{M}(T)$-set. In particular, $D_{y}$ is an independent majority dominating set of $T_{y}$ and so, $\left|D_{y}\right|=\gamma_{M}\left(T_{y}\right) \leq i_{M}\left(T_{y}\right) \leq\left|D_{y}\right|$. Hence $\left|D_{y}\right|=i_{M}\left(T_{y}\right)$ and $D_{y}$ is a $i_{M}\left(T_{y}\right)$-set. Thus, every $\gamma_{M}\left(T_{y}\right)$-set is an $i_{M}\left(T_{y}\right)$-set. $\therefore \gamma_{M}\left(T_{y}\right) \equiv i_{M}\left(T_{y}\right)$

Conversely, Let $\gamma_{M}\left(T_{y}\right) \equiv i_{M}\left(T_{y}\right) \ldots . . .(2)$. To prove $\gamma_{M}(T) \equiv i_{M}(T)$. Let $D$ be a $\gamma_{M}(T)$-set and $D_{y}=D \cap V\left(T_{y}\right)$ and $D_{w}=D \cap V\left(T_{w}\right) . \quad$ Suppose $w \notin D, \quad$ then $\left|D_{w}\right|=\left|S_{w}\right|$ and $\left|D_{y}\right|=\left|D-D_{w}\right|=|D|-\left|S_{w}\right| . \quad$ Then $\left|D_{y}\right|=\gamma_{M}(T)-\left|S_{w}\right| \Rightarrow \gamma_{M}(T)=\gamma_{M}\left(T_{y}\right)+\left|S_{w}\right|$, which is a contradiction to lemma(3.1), $\gamma_{M}(T)=\gamma_{M}\left(T_{y}\right)+1$. Hence $w \in D$. Then $D_{w}=\{w\} \in T_{w}$, since $w$ is a majority dominating vertex of $T_{w}$. Since $T$ has an edge $w y, w$ is the onlyvertex that dominates $y$. 
Since $y$ is already dominated by $w \in D_{w}, D_{y}$ does not contain $y$ in $T_{y}$. But $D_{y}$ is itself a majority dominating set of $T_{y}$ of $\left|D-D_{w}\right|$. ie., $\left|D_{y}\right|=\left|D-D_{w}\right|=|D|-1=\gamma_{M}(T)-1$. By lemma (3.1), $\left|D_{y}\right|=\gamma_{M}\left(T_{y}\right)$, by (2), $\left|D_{y}\right| \equiv i_{M}\left(T_{y}\right) \Rightarrow D_{y}$ is an independent majority dominating set of $T_{y}$. Furthermore, $D_{w}=\{w\}$ is also an independent majority dominating set of $T_{w}$. Hence $D$ is an $i_{M}(T)$-set. Thus every $\gamma_{M}(T)$-set is an $i_{M}(T)$ set. $\therefore \gamma_{M}(T) \equiv i_{M}(T)$.

Next, a construction for characterization of the trees $T$ for which $\gamma_{M}(T) \equiv i_{M}(T)$ is provided by using the following operation.

Operation -A: Let $w$ be a vertex of a tree $T_{w}$ such that every leaf of $T_{w}$, except possibly for $w$ itself, is at distance two from $w$. Let $S_{w}$ be the set of support vertices of $T_{w}$. Let $y$ be a pendant vertex of a nontrivial tree $T_{y}$. Let $T$ be obtained from $T_{w} \cup T_{y}$ by adding the edge $w y$. Define the family as $\mathfrak{I}_{1}=\left\{T / T=K_{1}\right.$ or Tis obtained fromanon-trivial star by a finite sequence of operation $\left.A\right\}$

\section{Theorem 3.3:}

For any tree $T, \gamma_{M}(T) \equiv i_{M}(T)$ if and only if $T \in \mathfrak{I}_{1}$

Proof: Let $T \in \mathfrak{J}_{1}$.If $T=K_{1}$ or if $T$ is a non-trivial star, then $\gamma_{M}(T)=i_{M}(T)=1$ and $\gamma_{M}(T) \equiv i_{M}(T)$. On the other hand,if $T$ is constructed from a non-trivial star by a finite sequence of atleast one operation(A), then repeated applications oflemma (3.2), we get $\gamma_{M}(T) \equiv i_{M}(T)$, since a star has majority domination number strongly equal to its independent majority domination number. Conversely, let $\gamma_{M}(T) \equiv i_{M}(T)$. To prove $T \in \mathfrak{I}_{1}$. By induction on the order $p$ of a tree $T$ for which $\gamma_{M}(T) \equiv i_{M}(T)$. If $T=K_{1}$ or $K_{2}$, then $T \in \mathfrak{I}_{1}$. If $\operatorname{diam} T=2$ then $T$ is a non-trivial star and so $T \in \mathfrak{I}_{1}$. When $\operatorname{diam} T=3,4,5,6$ which satisfy $\gamma_{M}(T) \equiv i_{M}(T)$ since $\gamma_{M}(T)=1=i_{M}(T)$. Then $T \in \mathfrak{I}_{1}$. Now, assume that $\operatorname{diam} T \geq 7$ which satisfy $\gamma_{M}(T) \equiv i_{M}(T)$. We now root the tree at a leaf $r$ of maximum eccentricity $\operatorname{diam} T$. Let $w$ be the vertex at distance $(\operatorname{diam} T-2)$ from $r$ on a longest path starting at $r$.

Let $T_{w}$ be the subtree of $T$ rooted at $w$. Then the vertex cannot be adjacent to a leaf. If not, it will contradict our assumption that $\gamma_{M}(T) \equiv i_{M}(T)$. Hence every leaf of $T_{w}$, except possibly for $w$ itself, is at distance two from $w$. Let $y$ denote the parent of $w$ on $T$ and let $T_{y}$ denote the component of $T-w y$ containing $y$. Since diam $T \geq 7, T_{y}$ is a non-trivial tree. By lemma (3.2), if $\gamma_{M}(T) \equiv i_{M}(T)$ then $\gamma_{M}\left(T_{y}\right) \equiv i_{M}\left(T_{y}\right)$.

Now, since $T_{y}$ is a tree of order less than $p$ satisfying $\gamma_{M}\left(T_{y}\right) \equiv i_{M}\left(T_{y}\right)$, we can apply the induction hypothesis, to $T_{y}$ to show that $T_{y} \in \mathfrak{I}_{1}$. Since $T$ is obtained form $T_{y}$ by a operation A, we have $T \in \mathfrak{I}_{1}$. Hence the theorem. $\square$

Theorem 3.4: Let $D_{i}$ be the set of all $\gamma_{M}$-sets of $G$. Then

(i). $\gamma_{M}(G) \equiv i_{M}(G)$ if and only if induced subgraph $\langle D\rangle$ has only isolates, for every

$$
\gamma_{M} \text {-set } D \in D_{i} \text {. }
$$

(ii). $\gamma_{M}(G)$ is not $\equiv i_{M}(G)$ if and only if the induced subgraph $\langle D\rangle$ is not totally disconnected for any

$\gamma_{M}$ - set $D \in D_{i}$.

Proof: Let $D_{i}$ be the set of all $\gamma_{M}$-set $D$ of a graph $G$.

(i). Suppose $\gamma_{M}(G) \equiv i_{M}(G)$. Then $\gamma_{M}(G) \leq i_{M}(G)$ and every $\gamma_{M}$-set $D$ of a graph $G$ is an independent majority dominating set of $G$. The induced subgraph $\langle D\rangle$ has only isolates for every $\gamma_{M}$-set 
$D \in D_{i}$. Conversely, for every $\gamma_{M}$-set $D$, the induced subgraph $\langle D\rangle$ has only isolates. Then $D$ is an independent set of $G \Rightarrow$ every $\gamma_{M}$-set $D$ is an $i_{M}$-set of $G . \therefore i_{M}(G) \leq \gamma_{M}(G)$. For any graph $G$, $\gamma_{M}(G) \leq i_{M}(G)$. Hence $\gamma_{M}(G) \equiv i_{M}(G)$.

(ii). Suppose $\gamma_{M}(G)$ is not $\equiv i_{M}(G)$. Then for any graph $G, \gamma_{M}(G) \leq i_{M}(G)$ but not every $\gamma_{M}$-set $D$ is an $i_{M}$-set of $G$. Then the $\gamma_{M}$-set $D$ is not independent for any one $D \in D_{i}$. Hence, the induced subgraph $\langle D\rangle$ is not totally disconnected for any $D \in D_{i}$. Conversely, if $\langle D\rangle$ is not totally disconnected for atleast one $D \in D_{i}$ then $D$ is not an independent $\gamma_{M}$-set. It does not satisfy the fact that every $\gamma_{M}$-set is an $i_{M}$-set of $G . \therefore i_{M}(G) \leq \gamma_{M}(G)$ is not true. Thus, $\gamma_{M}(G)$ is not $\equiv i_{M}(G)$.

\section{Strong Equality of $\gamma(G)$ and $\gamma_{M}(G)$ and of $i(G)$ and $i_{M}(G)$}

\section{Observations 4.1:}

1. For any graph $G, \gamma_{M}(G) \leq \gamma(G)$.

2. If $\gamma(G)=1$ then $\gamma_{M}(G)=1$

3. If $G$ has a full degree vertex then every $\gamma_{M}$-set is a $\gamma$-set.

\section{Proposition 4.2:}

For any graph $G, \gamma(G) \equiv \gamma_{M}(G)$ if and only if $G$ has a full degree vertex.

Proof: Let $\gamma(G) \equiv \gamma_{M}(G)$. Suppose $G$ has no full degree vertex. Then $\gamma(G) \geq 2$. $G$ may have a majority dominating vertex $v$ with $d(v) \geq\left\lceil\frac{p}{2}\right\rceil-1$. Then $\gamma_{M}(G)=1$ but $r>1$. Therefore every $\gamma_{M}$-set is not a $\gamma$ set $\Rightarrow \gamma(G)$ is not strongly equal to $\gamma_{M}(G)$, a contradiction. Hence $G$ has a full degree vertex. Conversely, if $G$ has a full degree vertex, then $r=1$. Then $\gamma_{M}(G)=1$. Since $\gamma(G)=\gamma_{M}(G)=1$, every $\gamma_{M}$-set is also a $\gamma$-set. Hence $\gamma(G) \equiv \gamma_{M}(G)$

\section{Proposition 4.3:}

For any graph $G, i(G) \equiv i_{M}(G)$ if and only if $G$ has a full degree vertex.

\section{References:}

[1]. J. JoselineManora, B. John, Independent Majority Dominating set of a Graph, International Journal of Applied Computational Science and Mathematics -Accepted.

[2]. J. JoselineManora, B. John, Majority Independence Number of a Graph, International Journal of Mathematical Research, Vol-6, No.1 (2014), 65-74

[3]. J. JoselineManora, V. Swaminathan, Majority dominating sets in graphs, Jamal Academic Research Journal, Vol-3, No.2 (2006), $75-82$.

[4]. J. JoselineManora, V. Swaminathan, Results on Majority dominating set, Science Magna, North West University, X'tion, P.R. china, Vol.7, No.3 (2011), 53-58

[5]. T. W. Haynes, M. A. Henning, P. J. Slater, Strong equality of domination parameters in trees, Discrete Mathematics 260 (2003) 77 87.

[6]. T. W. Haynes, S. T. Hedetniemi and P. J. Slater, Fundamentals of Domination in Graphs, Marcel Dekker, New York, 1998. 